\title{
CENTERS ON CENTER MANIFOLDS IN THE LÜ SYSTEM
}

\author{
ADAM MAHDI $^{1,2}$, CLAUDIO PESSOA $^{3}$, AND DOUGLAS S. SHAFER $^{2}$
}

\begin{abstract}
We confirm a conjecture of Mello and Coelho [Physics Letters A 373 (2009) 1116-1120] concerning the existence of centers on local center manifolds at equilibria of the Lü system of differential equations on $\mathbb{R}^{3}$. Our proof shows that the local center manifolds are algebraic ruled surfaces, and are unique.
\end{abstract}

\section{INTRODUCTION}

The Lü system is the system of nonlinear ordinary differential equations on $\mathbb{R}^{3}$ given by

$$
\begin{aligned}
& \dot{x}=P(x, y, z)=a(y-x) \\
& \dot{y}=Q(x, y, z)=c y-x z \\
& \dot{z}=R(x, y, z)=-b z+x y,
\end{aligned}
$$

where $a, b$, and $c$ are real parameters. This system has an equilibrium at the origin for all values of the parameters and a pair of symmetrically located equilibria $Q_{ \pm}=( \pm \sqrt{b c}, \pm \sqrt{b c}, c)$ when $b c>0$. For parameter values in the surface $\mathcal{S}=$ $\{(a, b, c): a b>0, \quad c=(a+b) / 3\}$ the matrix of first partial derivatives of the right hand sides of $(1)$ at $Q_{+}$and $Q_{-}$has two purely imaginary eigenvalues and one real eigenvalue, which has opposite sign from $a+b$. It was shown in [3] that for parameter values in the straight line $\mathcal{C}=\{(a, b, c): a \neq 0, \quad b=2 c, \quad c=a\}$ in $\mathcal{S}$ the first three Lyapunov quantities at $Q_{ \pm}$are zero, which led the authors of that letter to conjecture the following result, which they described as unexpected.

Conjecture ([3]). For parameter values in $\mathcal{C}$ satisfying $a+b \neq 0$ the equilibria $Q_{ \pm}$are nonlinear centers for the flow of system (1) restricted to the local center manifolds.

In this letter we prove that the Conjecture is true and in the process unfold some of the structure of the local center manifolds.

\section{Proof of the Conjecture}

System (1) is invariant under the involution $(x, y, z) \rightarrow(-x,-y, z)$, so we may confine our attention to $Q_{+}$. By the Center Manifold Theorem (see for example [4]) there is a two-dimensional invariant manifold $W^{c}$ in a neighborhood of $Q_{+}$ that is tangent to the center eigenspace at $Q_{+}$and contains all the local recurrent behavior. But although system (1) is polynomial, a priori $W^{c}$ need not be even $C^{\infty}$, and it need not be unique. Our first attempt at a proof of the Conjecture is

2010 Mathematics Subject Classification. 34C40, 34C15, 34C60, 37C10.

Key words and phrases. Center problem, center manifold, integrability. 
to try to avoid the center manifold altogether by means of the following theorem of Lyapunov ([2]). A proof may be found in [1, §13].

Theorem (Lyapunov Center Theorem). Suppose $U$ is an open neighborhood of the origin in $\mathbb{R}^{3}$, $\mathbf{f}: U \rightarrow \mathbb{R}^{3}$ is a real analytic mapping, and that $d \mathbf{f}(\mathbf{0})$ has one non-zero and two pure imaginary eigenvalues. By an invertible linear change of coordinates and a possibly negative rescaling of time place the system of differential equations $\dot{\mathbf{u}}=\mathbf{f}(\mathbf{u})$ in the form

$$
\begin{aligned}
\dot{u} & =P(u, v, w)=-\omega v+\widetilde{P}(u, v, w) \\
\dot{v} & =Q(u, v, s)=\omega u+\widetilde{Q}(u, v, w) \\
\dot{w} & =R(u, v, w)=-\lambda w+\widetilde{R}(u, v, w),
\end{aligned}
$$

where $\omega$ and $\lambda$ are positive real numbers. Let $\mathfrak{X}$ denote the corresponding vector field

$$
\mathfrak{X}=P \frac{\partial}{\partial u}+Q \frac{\partial}{\partial v}+R \frac{\partial}{\partial w}
$$

on a neighborhood of the origin, and let $W^{c}$ denote a local center manifold at the origin. Then the origin is a center for $\mathfrak{X} \mid W^{c}$ if and only if $\mathfrak{X}$ admits a real analytic local first integral of the form $\Phi(u, v, w)=u^{2}+v^{2}+\cdots$ in a neighborhood of the origin in $\mathbb{R}^{3}$.

We have abused notation slightly so that $\mathfrak{X}$ given by (3) can stand for the vector field that corresponds to the Lü system written either in its original form (1) or in the form (2).

One technique for identifying first integrals for any polynomial vector field $\mathfrak{X}$ is the following method of Darboux. Given a polynomial $F(x, y, z)$ the algebraic surface with equation $F(x, y, z)=0$ is invariant for $\mathfrak{X}$ if and only if there exists a polynomial $K(x, y, z)$, the cofactor of $F$, such that $\mathfrak{X} F=K F$. If there exists some number $n$ of pairs $\left(F_{j}, K_{j}\right)$ for which there exists a nontrivial dependency relation $\sum \alpha_{j} K_{j}=0$ then $F_{1}^{\alpha_{1}} \cdots F_{n}^{\alpha_{n}}$ is a first integral for $\mathfrak{X}$.

As a first step in the proof proper, by a translation we move $Q_{+}$to the origin, then by a linear change of coordinates place the linear part of $\mathfrak{X}$ in real Jordan normal form. This and a time rescaling ultimately yields from (1)

$$
\begin{aligned}
& \dot{x}=24 \sqrt{2} a y+4 x^{2}+4 \sqrt{2} x y-2 \sqrt{2} y z-z^{2} \\
& \dot{y}=-24 \sqrt{2} a x-16 \sqrt{2} x^{2}-8 x y-6 \sqrt{2} x z+4 y z+7 \sqrt{2} z^{2} \\
& \dot{z}=-48 a z+8 x^{2}+8 \sqrt{2} x y-4 \sqrt{2} y z-2 z^{2} .
\end{aligned}
$$

For system (4) it can be shown that there is exactly one algebraic invariant surface, given by

$$
F(x, y, z)=4 x^{2}-4 x z+z^{2}-24 a z=0 .
$$

This is inadequate for construction of a first integral, but we observe that $F(x, y, z)=$ 0 is tangent to the center eigenspace, the $(x, y)$-plane, at the origin, hence in a neighborhood of the origin forms a local center manifold, which we denote $W^{c}$.

The absence of $y$-dependence in (5) suggests that $\mathfrak{X} \mid W^{c}$ might possess a timereversible symmetry, a common mechanism for existence of a center, but this is not the case. Indeed, solving (5) for $z$ and inserting into the first two equations in (4) 
we obtain for $\mathfrak{X} \mid W^{c}$ in local coordinates

$$
\begin{aligned}
& \dot{x}=-288 a^{2}-96 a x \\
& +96 a \sqrt{3 a(3 a+x)}+16 x \sqrt{3 a(3 a+x)}+8 y \sqrt{6 a(3 a+x)} \\
& \dot{y}=2016 \sqrt{2} a^{2}+576 \sqrt{2} a x+48 a y \\
& -672 a \sqrt{6 a(3 a+x)}-88 x \sqrt{6 a(3 a+x)}-16 y \sqrt{3 a(3 a+x)}
\end{aligned}
$$

which is not invariant under the involution $(x, y, t) \rightarrow(x,-y,-t)$.

To return to the category of polynomial systems of differential equations in which the Darboux theory and other techniques are applicable we make the change of coordinates

$$
u^{2}=12 a x+36 a^{2}, \quad y=y,
$$

which is valid on a neighborhood of the origin and which shifts the equilibrium of interest to $(u, y)=(6|a|, 0)$. Translating the equilibrium to the origin and rescaling we obtain the system

$$
\begin{aligned}
& \dot{u}=72 \sqrt{2} a^{2} y+12 a u^{2} \\
& \dot{y}=-72 \sqrt{2} a^{2} u+54 \sqrt{2} a u^{2}-24 a u y-11 \sqrt{2} a u^{3} .
\end{aligned}
$$

But this system is Hamiltonian with Hamiltonian function

$$
H(u, y)=-\sqrt{2}\left(36 a^{2} u^{2}-18 a u^{3}+\frac{11}{4} u^{4}\right)-12 a u^{2} y-36 \sqrt{2} a^{2} y^{2} .
$$

Thus the equilibrium at the origin, which must be a focus or a center, is in fact a center, as was to be shown.

\section{Conclusion}

We have verified the conjecture of Mello and Coelho that the isolated equilibria $Q_{+}$and $Q_{-}$remote from the origin in system (1) when $b c>0$ are centers for the flow restricted to local center manifolds at those points, when $a+b \neq 0$. It is known that in this circumstance the local center manifolds are unique (for example, Theorem 3.2 in [4]). Our proof shows that the local center manifolds are algebraic ruled surfaces, which by reversing the various coordinate transformations can be written explicitly. Moreover the same algebraic surface contains both $Q_{+}$and $Q_{-}$and is the only invariant algebraic surface in the state space. This fact is illustrated in the abstract representation of the phase portrait shown in Figure 1. The existence of the homoclinic loops bounding the centers is proved by examining the Hamiltonian function. A computer plot of trajectories near each center and the figure eight is shown in Figure 2. The orientation of this figure is a quarter turn counterclockwise from that of the Figure 1.

It is a consequence of the Lyapunov Center Theorem that the Lü system admits an analytic first integral in a neighborhood of $Q_{+}$and $Q_{-}$, although neither the integral nor its domain of definition are known explicitly. 


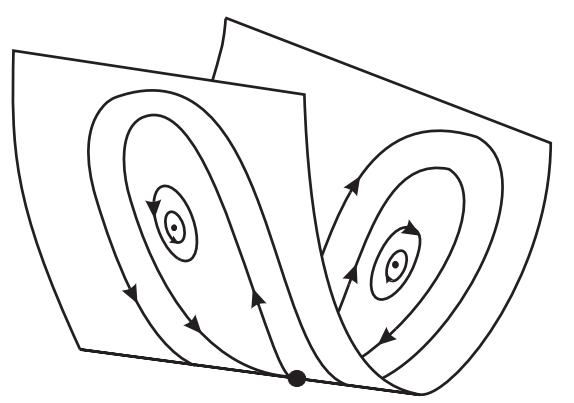

FiguRE 1. The phase portrait on the invariant ruled surface that contains the center manifolds at $Q_{+}$and $Q_{-}$.

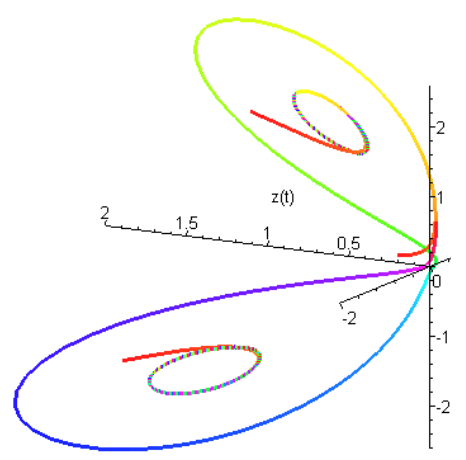

FiguRE 2. Numerical plot of trajectories near each center and the figure eight for parameter values $a=1, b=2$, and $c=1$. Initial conditions: $(0,0.1,0.2),(\sqrt{2}, 1.41421,1.5),(-\sqrt{2},-1.41421,1.5)$.

\section{REFERENCES}

[1] Bibikov, Y. N. Local Theory of Nonlinear Analytic Ordinary Differential Equations. Lecture Notes in Mathematics, Vol. 702. New York: Springer-Verlag, 1979.

[2] Liapounoff, M. A. Problème général de la stabilité du mouvement. Annales de la Faculté des Sciences de Toulouse Sér. 29 (1907) 204-474. Photo-reproduction in Annals of Mathematics Studies 17, Princeton: Princeton University Press, 1947, reprinted 1965, Kraus Reprint Corporation, New York.

[3] Mello, L. F., and S. F. Coelho. Degenerate Hopf bifurcations in the Lü system. Physics Letters A 373 (2009) 1116-1120.

[4] Sijbrand, J. Properties of center manifolds. Trans. Amer. Soc. 289 (1985) 431-469.

1 Mathematics Department, University of North Carolina at Charlotte, Charlotte, NORTh CAROLINA 28223, USA

E-mail address: adam.mahdi@uncc.edu, dsshafer@uncc.edu

${ }^{2}$ Faculty of Applied Mathematics, AGH University of Science and Technology, al. Mickiewicza 30, 30-059 Kraków, Poland

3 Universidade Estadual Paulista, IBIlce-Unesp, Av. Cristovão Colombo, 2265 15.054-000, S.J. Rio Preto, SP, Brasil

E-mail address: pessoa@ibilce.unesp.br 